J. Math. Soc. Japan

Vol. 20, Nos. 1-2, 1968

\title{
Pluricanonical systems on algebraic surfaces of general type
}

\author{
Dedicated to Professor S. Iyanaga on his 60 th birthday
}

\author{
By Kunihiko KODAIRA
}

(Received Aug. 10, 1967)

By a minimal non-singular algebraic surface of general type we shall mean a non-singular algebraic surface free from exceptional curves (of the first kind) of which the bigenus $P_{2}$ and the Chern number $c_{1}^{2}$ are both positive, where $c_{1}$ denote the first Chern class of the surface (see $\S 3$ ). Let $S$ denote a minimal non-singular algebraic surface of general type defined over the field of complex numbers and let $K$ be a canonical divisor on $S$. The number of non-singular rational curves $E$ on $S$ satisfying the equation: $K E=0$ is smaller than the second Betti number of $S$, where $K E$ denotes the intersection multiplicity of $K$ and $E$. We define $\mathcal{E}$ to be the union of all the non-singular rational curves $E$ with $K E=0$ on $S$ and represent it as a sum $: \mathcal{E}=\sum_{\nu} \mathcal{E}_{\nu}$ of its connected components $\mathcal{E}_{\nu}$. Obviously $\mathcal{E}$ may be an empty set. Consider a holomorphic map $\Phi: z \rightarrow \Phi(z)$ of $S$ into a projective $n$-space $\boldsymbol{P}^{n}$. We shall say that $\Phi$ is biholomorphic modulo $\mathcal{E}$ if and only if $\Phi$ is biholomorphic on $S-\mathcal{E}$ and $\Phi^{-1} \Phi(z)=\mathcal{E}_{\nu}$ for $z \in \mathcal{E}_{\nu}$. For any positive integer $m$, we let $\Phi_{m K}$ denote the rational map of $S$ into $\boldsymbol{P}^{n}$ defined by the pluri-canonical system $|m K|$, where $n=\operatorname{dim}|m K|$. Note that, if $|m K|$ has no base point, then $\Phi_{m K}$ is a holomorphic map. D. Mumford proved that, for every sufficiently large integer $m$, the pluri-canonical system $|m K|$ has no base point and $\Phi_{m K}$ is biholomorphic modulo $\mathcal{E}$ (see Mumford [6]; compare also Zariski [9], Matsusaka and Mumford [5]). His proof is based on results of Zariski. [9] and covers the abstract. case. On the other hand, it has been shown by Šafarevic [8] that $\Phi_{9 K}$ is a birational map. The main purpose of this paper is to prove the following: theorem :

THEOREM. For every integer $m \geqq 4$, the pluri-canonical system $|m K|$ has no base point and $\Phi_{m K}$ is a holomorphic map. For every integer $m \geqq 6$, the map. $\Phi_{m K}$ is biholomorphic modulo $\mathcal{E}$. 


\section{$\S 1$. Notation.}

Let $S$ be a non-singular algebraic surface defined over the field $C$ of complex numbers. We shall denote by $x, y, z$ points on $S$, by $C, C_{1}, \cdots, \Theta, \cdots$ irreducible curves on $S$, by $X, Y, D, D_{1}, \cdots$ divisors on $S$ and by $m, n, h, i, j, k$ rational integers. We say that a divisor $D=\sum_{i} n_{i} C_{i}$ is positive and write $D>0$ if the coefficients $n_{i}$ are positive. For any divisors $D$ and $X$ on $S$ we denote by $D X$ the intersection multiplicity of $D$ and $X$. We write $D^{2}$ for $D D$. We indicate by the symbol $\approx$ linear equivalence. We let $[D]$ denote the complex. line bundle over $S$ determined by the divisor $D$.

Let $F$ be a complex line bundle over $S$. By a local holomorphic section of $F$ we shall mean a holomorphic section of $F$ defined over an open subset of $S$. Let $\varphi: z \rightarrow \varphi(z)$ be a local holomorphic section of $F$. We choose a sufficiently fine finite covering $\left\{U_{j}\right\}$ of $S$ and denote by $\varphi_{j}(z)$ the fibre coordinate of $\varphi(z)$ over $U_{j}$, provided that $z \in U_{j}$. Let $x$ be a point on $S$ and let $\left(z_{1}, z_{2}\right)$ denote a local coordinate of the center $x$ on $S$. We call $x$ a zero of $\varphi$ of order $h$ if

$$
\varphi_{j}(x)=0, \quad\left(\partial^{m \vdash n} \varphi_{j} / \partial z_{1}{ }^{m} \partial z_{2}{ }^{n}\right)(x)=0 \quad \text { for } m+n \leqq h-1
$$

and if at least one partial derivative $\left(\partial^{h} \varphi_{j} / \partial z_{1}{ }^{n} \partial z_{2}{ }^{h-n}\right)(x)$ of order $h$ does not vanish, provided that $x \in U_{j}$. We denote by $\mathcal{O}$ the sheaf over $S$ of germs of holomorphic functions and by $\mathcal{O}(F)$ the sheaf over $S$ of germs of holomorphic sections of $F$. Moreover we denote by the symbol

$$
\mathcal{O}(F-h x-k y-\cdots)
$$

the subsheaf of $\mathcal{O}(F)$ consisting of germs of those holomorphic sections of $F$ of which the points $x, y, \cdots$ are zeros of respective orders $\geqq h, \geqq k, \cdots$. We remark that $\mathcal{O}(-x)$ is the sheaf of the ideals of the point $x$ and that

$$
\mathcal{O}(F-h x-k y-\cdots)=\mathcal{O}(F) \otimes_{\mathcal{O}} \mathcal{O}(-x)^{h} \mathcal{O}(-y)^{k} \cdots .
$$

Let $\boldsymbol{C}^{n}$ denote the vector space of $n$ complex variables. The stalks of the quotient sheaf $\mathcal{O} / \mathcal{O}(-x)^{h}$ are

$$
\left(\mathcal{O} / \mathcal{O}(-x)^{h}\right)_{z}=\left\{\begin{array}{l}
C^{h(h+1) / 2}, \\
0
\end{array}\right.
$$

To indicate this we write

$$
C_{x}^{h(h+1) / 2}=\mathcal{O} / \mathcal{O}(-x)^{h} .
$$

Then, for instance, we have

$$
\mathcal{O}(F) / \mathcal{O}(F-h x-k y) \cong \boldsymbol{C}_{x}^{h(h+1) / 2} \oplus \boldsymbol{C}_{y}^{h(k+1) / 2} .
$$

For any holomorphic section $\phi$ of a complex line bundle over $S$, we denote 
by $(\phi)$ the divisor of $\phi$. Let $D$ be a positive divisor on $S$. Obviously $D$ is the divisor $(\psi)$ of a holomorphic section $\psi$ of the complex line bundle $[D]$. We say that $x$ is a point of $D$ and write $x \in D$ if and only if $x$ is a zero of $\phi$. We define the multiplicity of a point $x$ of $D$ to be $m$ if $x$ is a zero of $\psi$ of order $m$. Moreover we call $x$ a simple point or a multiple point of $D$ according as $m=1$ or $m \geqq 2$. We shall say that a local holomorphic section $\varphi$ of $F$ defined on an open subset $W \subset S$ is divisible by $D$ if $\varphi_{j} / \psi_{j}$ is holomorphic on $U_{j} \cap W$ for every neighborhood $U_{j}$. We denote by $\mathcal{O}(F-D)$ the sheaf over $S$ of germs of those holomorphic sections of $F$ which are divisible by $D$. We have the isomorphism:

$$
\mathcal{O}(F-D) \cong \mathcal{O}(F-[D])
$$

We define

$$
\mathcal{O}(F-D-h x-k y-\cdots)=\mathcal{O}(F-D) \cap \mathcal{O}(F-h x-k y-\cdots) .
$$

Note that, if $x$ is a point of $D$ of multiplicity $m \geqq h$, then

$$
\mathcal{O}(F-D-h x-k y-\cdots)=\mathcal{O}(F-D-k y-\cdots) \text {. }
$$

We denote by $|F|$ the complete linear system consisting of the divisors $(\varphi)$ of holomorphic sections $\varphi \in H^{0}(S, \mathcal{O}(F)), \varphi \neq 0$, and define

$$
\operatorname{dim}|F|=\operatorname{dim} H^{0}(S, \mathcal{O}(F))-1 \text {. }
$$

Note that $|[D]|=|D|$. Letting $\left\{\varphi_{0}, \varphi_{1}, \cdots, \varphi_{n}\right\}$ be a base of the linear space $H^{\circ}(S, \mathcal{O}(F))$, we define a rational map

$$
\Phi_{F}: z \rightarrow \Phi_{F}(z)=\left(\varphi_{0}(z), \varphi_{1}(z), \cdots, \varphi_{n}(z)\right)
$$

of $S$ into $\boldsymbol{P}^{n}$. We call $z$ a base point of the complete linear system $|F|$ if $z \in D$ for all divisors $D \in|F|$. It is obvious that, if $|F|$ has no base point, then $\Phi_{F}$ is a holomorphic map. We let $K$ denote either the canonical bundle of $S$ or a canonical divisor on $S$. We denote by $p_{g}, P_{m}$ and $q$, respectively, the geometric genus, the $m$-genus and the irregularity of $S$. Note that

$$
P_{m}=\operatorname{dim}|m K|+1, \quad m=1,2,3, \cdots .
$$

For any divisor $X$ on $S$ we let $\pi(X)$ denote the virtual genus of $X$ defined by the formula:

$$
2 \pi(X)-2=X^{2}+K X
$$

Every complex line boundle $F$ over $S$ is determined by a divisor $D$ on $S: F$ $=[D]$. We let $F^{2}=D^{2}$. Moreover, for any divisor $X$ on $S$, we define

$$
F X=D X, \quad F=[D] .
$$




\section{§2. Vanishing theorems.}

Let $F$ be a complex line bundle over $S$ and let $C$ denote an irreducible curve on $S$. We define the restriction to $C$ of the sheaf $\mathcal{O}(F)$ to be the quotient sheaf :

$$
\mathcal{O}(F)_{C}=\mathcal{O}(F) / \mathcal{O}(F-C) .
$$

For any element $\varphi$ of $\mathcal{O}(F)$ we denote by $\varphi_{C}$ the element of $\mathcal{O}(F)_{C}$ corresponding to $\varphi$.

Let $\tilde{C}$ denote the non-singular model of $C$ and let $\mu$ be the holomorphic birational map of $\tilde{C}$ onto $C$. Moreover let $\mu^{*} F$ denote the complex line bundle over $\tilde{C}$ induced from $F$. For any complex line bundle $f$ over $\tilde{C}$ we denote by $c(\mathfrak{f})$ the Chern class of $\mathfrak{f}$ which can be regarded as an integer. We have

$$
c\left(\mu^{*} F\right)=F C \text {. }
$$

Letting $\delta$ be an effective divisor on $\widetilde{C}$, we denote by $\mathcal{O}(\mathfrak{f}-\delta)$ the sheaf over $\tilde{C}$ of germs of holomorphic sections of $\mathfrak{f}$ which are divisible by $\delta$. Let $\mathfrak{c}$ denote the conductor of $C$ on $\tilde{C}$. We have the exact sequence

$$
0 \longrightarrow \mathcal{O}\left(\mu^{*} F-\mathfrak{c}\right) \stackrel{\mu}{\longrightarrow} \mathcal{O}(F)_{C} \longrightarrow M \longrightarrow 0,
$$

where $M$ is a sheaf over $C$ such that the stalk $M_{z}$ is zero for every simple point $z$ of $C$. In forming the exact sequence (3) we regard $\mathcal{O}\left(\mu^{*} F-c\right)$ as a sheaf over $C$ by means of the map $\mu: \widetilde{C} \rightarrow C$ (see [2], $\S 1$ ).

In what follows we denote by $\boldsymbol{C}\{t\}$ the ring of convergent power series in a variable $t$ with coefficients in $C$. Let $x$ be a point of $C$ of multiplicity $m$. The inverse image $\mu^{-1}(x)$ consists of a finite number of points $p_{1}, \cdots, p_{\lambda}, \cdots, p_{r}$ on $\tilde{C}$. We introduce a local coordinate $(w, z)$ of the center $x$ on $S$ which is "general" with respect to $C$ (we write $w, z$ in place of $z_{1}, z_{2}$ ). Then, for each point $p_{\lambda}$, we find a local uniformization variable $t_{\lambda}$ of the center $p_{\lambda}$ on $\tilde{C}$ such that, in a neighborhood of $p_{\lambda}$, the map $\mu$ takes the following form

$$
\mu: t_{\lambda} \rightarrow(w, z)=\left(P_{\lambda}\left(t_{\lambda}\right), t_{\lambda}^{m_{\lambda}}\right), \quad P_{\lambda}\left(t_{\lambda}\right) \in t_{\lambda}^{m_{\lambda}} \boldsymbol{C}\left\{t_{\lambda}\right\},
$$

where $m_{\lambda}$ is a positive integer and $t_{\lambda}^{m} \lambda \boldsymbol{C}\left\{t_{\lambda}\right\}$ denotes the ideal of $\boldsymbol{C}\left\{t_{\lambda}\right\}$ generated by $t_{\lambda}^{m}$. It is clear that

$$
R(w, z)=\prod_{\lambda=1}^{r} \prod_{k=0}^{m} \prod^{-1}\left(w-P_{\lambda}\left(\varepsilon_{\lambda}^{k} z^{1 / m \lambda}\right)\right), \quad \varepsilon_{\lambda}=e^{2 \pi i / m} \lambda,
$$

is a polynomial of the form

$$
w^{m}+A_{1}(z) w^{m-1}+\cdots+A_{m}(z), \quad A_{k}(z) \in z^{k} C\{z\},
$$

and the equation: 


$$
R(w, z)=w^{m}+A_{1}(z) w^{m-1}+\cdots+A_{m}(z)=0
$$

is a minimal equation of $C$ on a neighborhood of $x$. We let

We define

$$
B_{h}(w, z)=w^{h}+A_{1}(z) w^{h-1}+\cdots+A_{h}(z) .
$$

$$
\sigma_{\lambda} d t_{\lambda}=d\left(t_{\lambda}^{m} \lambda\right) / \partial_{w} R\left(P_{\lambda}\left(t_{\lambda}\right), t_{\lambda}^{m_{\lambda}}\right),
$$

where $\partial_{w} R(w, z)=\partial R(w, z) / \partial w$. The exponent $c_{\lambda}$ in the expansion

$$
\sigma_{\lambda}=t_{\lambda}^{-c \lambda}\left(a_{\lambda_{0}}+a_{\lambda_{1}} t_{\lambda}+a_{\lambda 2} t_{\lambda}^{2}+\cdots\right), \quad a_{\lambda 0} \neq 0,
$$

is a non-negative integer and, by definition,

$$
\mathfrak{c}=c_{1} p_{1}+\cdots+c_{\lambda} p_{\lambda}+\cdots+c_{r} p_{r}+\cdots .
$$

Since the complex line bundle $F$ is locally trivial, the restriction to the point $x$ of the exact sequence (3) is reduced to

$$
0 \longrightarrow \bigoplus_{\lambda=1}^{r} \mathcal{O}(-\mathfrak{c})_{p_{\lambda}} \stackrel{\mu}{\longrightarrow}\left(\mathcal{O}_{C}\right)_{x} \longrightarrow M_{x} \longrightarrow 0 .
$$

For any convergent power series $f=f(w, z)$ in $w$ and $z$, we denote by $f_{C}$ the restriction of $f$ to $C$. Obviously the stalk $\left(\mathcal{O}_{C}\right)_{x}$ consists of the restrictions $f_{c}$ of elements $f$ of $\mathcal{O}_{x}$. It is clear that $\mathcal{O}(-\mathfrak{c})_{p_{\lambda}}=t_{\lambda}^{c} \boldsymbol{C}\left\{t_{\lambda}\right\}$. Hence an arbitrary element of the ring $\bigoplus_{\lambda=1}^{r} \mathcal{O}(-\mathrm{c})_{p_{\lambda}}$ can be written in the form

$$
\xi=\sum_{\lambda=1}^{r} \xi_{\lambda}\left(t_{\lambda}\right), \quad \xi_{\lambda}\left(t_{\lambda}\right) \in t_{\lambda}^{c} \lambda \boldsymbol{C}\left\{t_{\lambda}\right\} .
$$

LEMMA 1. For any element $\xi=\sum_{\lambda=1}^{r} \xi_{\lambda}\left(t_{\lambda}\right)$ of the ring $\underset{\lambda=1}{\oplus} \mathcal{O}(-\mathrm{c})_{p_{\lambda}}$, there exists one and only one element $f$ of $\mathcal{O}_{x}$ of the form

$$
f=\sum_{h=0}^{m-1} f_{h}(z) w^{m-1-h}, \quad f_{h}(z)=\sum_{n=0}^{\infty} f_{h n} z^{n},
$$

which satisfies the equation:

$$
f_{C}=\mu \xi \text {. }
$$

Moreover the coefficients $f_{h n}$ of $f$ are given by the formula

$$
f_{h n}=\frac{1}{2 \pi i} \sum_{\lambda=1}^{r} \oint \xi_{\lambda}\left(t_{\lambda}\right) B_{h}\left(P_{\lambda}\left(t_{\lambda}\right), t_{\lambda}^{m}\right) t_{\lambda}^{-(n+1) m_{\lambda}} \sigma_{\lambda} d t_{\lambda} .
$$

For a proof of this lemma, see [2], Appendix I.

For any integer $h$, we denote by $h^{+}$the positive part of $h$, i.e., $h^{+}$ $=\max \{h, 0\}$.

LEMMA 2. Let $k$ be a non-negative integer and let

$$
\mathfrak{๖}_{x}=\sum_{\lambda=1}^{r}(k-m+1)^{+} m_{\lambda} p_{\lambda} .
$$


Then we have

$$
\mu \bigoplus_{\lambda=1}^{r} \mathcal{O}\left(-\mathfrak{c}-\mathfrak{b}_{x}\right)_{p_{\lambda}} \subset\left(\mathcal{O}(-k x)_{c}\right)_{x} .
$$

PROOF. We take an arbitrary element $\xi$ of $\underset{\lambda}{\oplus} \mathcal{O}\left(-\mathfrak{c}-\mathfrak{b}_{x}\right)_{p_{\lambda}}$ and, with the aid of the above lemma, determine an element $f$ of $\mathcal{O}_{x}$ satisfying the equation: $f_{C}=\mu \xi$. Let $d_{\lambda}=(k-m+1)^{+} m_{\lambda}$. We then have

Since

$$
\xi=\sum_{\lambda=1}^{r} \xi_{\lambda}\left(t_{\lambda}\right), \quad \xi_{\lambda}\left(t_{\lambda}\right) \in t_{\lambda}^{c_{\lambda}+d_{\lambda}} \boldsymbol{C}\left\{t_{\lambda}\right\} .
$$

and

$$
\xi_{\lambda}\left(t_{\lambda}\right) B_{h}\left(P_{\lambda}\left(t_{\lambda}\right), t_{\lambda}^{m} \lambda\right) t_{\lambda}^{-(n+1) m_{\lambda}} \sigma_{\lambda} \in t_{\lambda}^{(h-n-1) m_{\lambda}+d_{\lambda} C} \boldsymbol{C}\left\{t_{\lambda}\right\}
$$

$$
(h-n-1) m_{\lambda}+d_{\lambda} \geqq 0 \quad \text { for } m-1-h+n \leqq k-1,
$$

we infer from (4) that

$$
f_{k n}=0, \quad \text { for } m-1-h+n \leqq k-1 .
$$

It follows that $f \in \mathcal{O}(-k x)_{x}$, q. e.d.

We remark that, in the case in which $x$ is a simple point of $C$, the formula (5) is reduced to the equality

$$
\mu \mathcal{O}\left(-\delta_{x}\right)_{p}=\left(\mathcal{O}(-k x)_{C}\right)_{x}, \quad p=\mu^{-1}(x) .
$$

THEOREM 1. Let $C$ be an irreducible curve on $S$ and let $F$ denote a complex line bundle over $S$. Moreover let $x$ and $y$ be distinct points of $C$ with respective multiplicities $m$ and $n$ and let $h$ and $k$ denote non-negative integers. If

$$
F C-C^{2}-K C>(h-m+1)^{+} m+(k-n+1)^{+} n,
$$

then the cohomology group $H^{1}\left(C, \mathcal{O}(F-h x-k y)_{c}\right)$ vanishes.

Proof. In view of Lemma 2 and the above remark, we have the exact sequence

$$
0 \rightarrow \mathcal{O}\left(\mu^{*} F-\mathfrak{c}-\mathfrak{b}_{x}-\mathfrak{b}_{y}\right) \rightarrow \mathcal{O}(F-h x-k y)_{C} \rightarrow M^{\prime \prime} \rightarrow 0,
$$

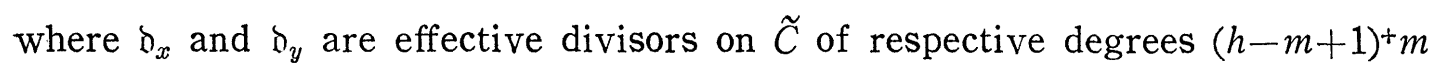
and $(k-n+1)^{+} n$ and $M^{\prime \prime}$ is a sheaf over $C$ such that the stalk $M_{z}^{\prime \prime}$ vanishes for every simple point $z$ of $C$. Hence we obtain the exact sequence

$$
\cdots \rightarrow H^{1}\left(\tilde{C}, \mathcal{O}\left(\mu^{*} F-\mathfrak{c}-\mathfrak{S}_{x}-\overleftarrow{\complement}_{y}\right)\right) \rightarrow H^{1}\left(C, \mathcal{O}(F-h x-k y)_{C}\right) \rightarrow 0 .
$$

Let denote the canonical bundle of $\tilde{C}$. Since

(see [2], § 2), we have

$$
\mathfrak{i}=\mu^{*}([C]+K)-[\mathrm{c}]
$$

$$
c\left(\mu^{*} F-\left[c+\delta_{x}+\delta_{y}\right]-\mathfrak{f}\right)=F C-C^{2}-K C-(h-m+1)^{+} m-(k-n+1)^{+} n>0 .
$$


Hence, using the duality theorem, we infer that

$$
H^{1}\left(\tilde{C}, \mathcal{O}\left(\mu^{*} F-\mathfrak{c}-\mathfrak{D}_{x}-\delta_{y}\right)\right)=0 .
$$

Combining this with the above exact sequence, we conclude that

q.e.d.

$$
H^{1}\left(C, \mathcal{O}(F-h x-k y)_{C}\right)=0,
$$

THEOREM 2. Let $F$ be a complex line bundle over $S$ with $F^{2}>0$. If there exists a positive integer $m$ such that the complete linear system $|m F|$ has no base point, then the cohomology group $H^{1}(S, \mathcal{O}(F+K))$ vanishes.

Proof. Let $\left\{\varphi_{0}, \varphi_{1}, \cdots, \varphi_{n}\right\}$ be a base of the linear space $H^{0}(S, \mathcal{O}(m F))$. Since, by hypothesis, $|m F|$ has no base point,

$$
\Phi: z \rightarrow \Phi(z)=\left(\varphi_{0}(z), \varphi_{1}(z), \cdots, \varphi_{n}(z)\right)
$$

is a holomorphic map of $S$ into a projective $n$-space $\boldsymbol{P}^{n}$. Suppose that the image $\Phi(S)$ is a curve in $\boldsymbol{P}^{n}$. Then, for any pair of general hyperplanes $L_{1}$ and $L_{2}$ in $\boldsymbol{P}^{n}$, the intersection $\Phi(S) \cap L_{1} \cap L_{2}$ is empty. The inverse images $D_{1}=\Phi^{-1}\left(L_{1}\right)$ and $D_{2}=\Phi^{-1}\left(L_{2}\right)$ are divisors belonging to $|m F|$. It follows that $m^{2} F^{2}=D_{1} D_{2}=0$. This contradicts that $F^{2}>0$. Thus we see that the image $\Phi(S)$ is a surface in $\boldsymbol{P}^{n}$.

Let $\left\{U_{j}\right\}$ be a finite covering of $S$ by small open subsets $U_{j}$. The complex line bundle $F$ is determined by a 1 -cocycle $\left\{f_{j k}\right\}$ composed of non-vanishing holomorphic functions $f_{j k}=f_{j k}(z)$ with respective domains $U_{j} \cap U_{k}$. Let $\varphi_{\lambda j}(z)$ denote the fibre coordinate of $\varphi_{\lambda}(z)$ over $U_{j}$ and let

$$
a_{j}(z)=\left(\sum_{\lambda=0}^{n}\left|\varphi_{\lambda j}(z)\right|^{2}\right)^{1 / m}, \quad \text { for } z \in U_{j} .
$$

Since $|m F|$ has no base point, $a_{j}(z)$ is positive. Moreover, since

we have

$$
\varphi_{\lambda j}(z)=f_{j k}(z)^{m} \varphi_{\lambda k}(z), \quad \text { on } U_{j} \cap U_{k},
$$

$$
a_{j}(z)=\left|f_{j k}(z)\right|^{2} a_{k}(z), \quad \text { on } U_{j} \cap U_{k}
$$

We let

$$
\gamma=\frac{i}{2 \pi} \sum_{\alpha, \beta=1}^{2} \gamma_{\alpha \beta}(z) d z^{\alpha} \wedge d \bar{z}^{\beta}=\frac{i}{2 \pi} \partial \bar{\partial} \log a_{j}(z), \quad i=\sqrt{-1},
$$

on each open set $U_{j} \subset S$. The real $d$-closed $(1,1)$-form $\gamma$ thus defined belongs to the Chern class $c(F)$ of $F$ (see [3], Lemma). The $(1,1)$-form $m \gamma$ is induced from a standard Kähler form on $\boldsymbol{P}^{n}$ by the holomorphic map $\Phi: S \rightarrow \boldsymbol{P}^{n}$, while the image $\Phi(S)$ is a surface. Consequently, there exists a proper analytic subset $N$ of $S$ such that the Hermitian matrix $\left(\gamma_{\alpha \beta}(z)\right)$ is positive definite for every point $z \in S-N$. Hence, applying a differential geometric method of [3], we infer that $H^{1}(S, \mathcal{O}(F+K)$ ) vanishes (see Mumford [7]). 


\section{§3. Composition series of pluri-canonical divisors.}

Let $S$ be a non-singular algebraic surface and let $K$ denote a canonical divisor on $S$.

Definition. We call $S$ a minimal non-singular algebraic surface of general type if and only if $S$ is free from exceptional curves (of the first kind) and

$$
P_{2}=\operatorname{dim}|2 K|+1 \geqq 1, \quad K^{2} \geqq 1 .
$$

We remark that, if $S$ is free from exceptional curves of the first kind and if either $P_{2}=0$ or $K^{2} \leqq 0$, then $S$ is one of the following five types of surfaces: projective plane, ruled surface, $K 3$ surface, abelian variety, elliptic surface (see [4], Enriques [1], Šafarevič [8]).

In what follows in this paper we let $S$ denote a minimal non-singular algebraic surface of general type.

By a divisorial cycle on $S$ we shall mean a linear combination $\Sigma r_{i} C_{i}$ of a finite number of irreducible curves $C_{i}$ on $S$ with rational coefficients $r_{i}$. We say that a divisorial cycle $\sum r_{i} C_{i}$ is positive if the coefficients $r_{i}$ are positive. We indicate by the symbol $\sim$ homology with respect to rational coefficients. For any divisorial cycles $\xi$ and $\eta$ on $S$ we denote by $\xi \eta$ the intersection multiplicity of $\xi$ and $\eta$. We write $\xi^{2}$ for $\xi \xi$. Since, by hypothesis, $K^{2} \geqq 1$, the following lemma is an immediate consequence of Hodge's index theorem (see Zariski $[9], \S 6)$ :

Lemma 3. Let $\zeta$ be a divisorial cycle on $S$. If $K \zeta=0$ and if $\zeta \nsim 0$, then $\zeta^{2}$ is negative.

In connection with this lemma, we note that every positive divisorial cycle on $S$ is not homologous to zero.

We have the inequality: $K C \geqq 0$ for every irreducible curve $C$ on $S$. Moreover the equality: $K C=0$ holds if and only if $C$ is a non-singular rational curve with $C^{2}=-2$ (see Mumford [6]). In fact, since, by hypothesis, $P_{2} \geqq 1$, the bicanonical system $|2 K|$ contains a positive divisor $D$. If $K C<0$, then $D C<0$ and therefore $C^{2}$ is a negative integer, while $C^{2}+K C=2 \pi(C)-2$. Hence $\pi(C)=0, C^{2}=-1$ and thus $C$ is an exceptional curve of the first kind. If $K C=0$, then, by Lemma 3, we have

$$
2 \pi(C)-2=C^{2}+K C=C^{2}<0 .
$$

This proves that $\pi(C)=0$ and $C^{2}=-2$.

THEOREM 3. The number of those irreducible curves $E$ on $S$ which satisfy the equation: $K E=0$ is smaller than the second Betti number $b_{2}$ of $S$.

Proof. Let $E_{1}, \cdots, E_{i}, \cdots, E_{n}$ be irreducible curves on $S$ such that $K E_{i}=0$. For our purpose it suffices to show that the curves $E_{i}$ are homologically inde- 
pendent. Assume a homology

Then we have

$$
\sum_{i=1}^{k} r_{i} E_{i} \sim \sum_{i=k+1}^{n} r_{i} E_{i}, \quad r_{i} \geqq 0
$$

$$
\left(\sum_{1}^{k} r_{i} E_{i}\right)^{2}=\left(\sum_{k+1}^{n} r_{i} E_{i}\right)^{2}=\sum_{i=1}^{k} \sum_{j=k+1}^{n} r_{i} r_{j} E_{i} E_{j} \geqq 0 .
$$

Hence we infer from Lemma 3 that the coefficients $r_{i}$ vanish, q.e.d.

We denote by $\mathcal{E}$ the sum of all the irreducible curves $E_{i}$ on $S$ satisfying $K E_{i}=0$ :

$$
\mathcal{E}=E_{1}+\cdots+E_{i}+\cdots+E_{b}, \quad b<b_{2} .
$$

Obviously the vanishing of $K E_{i}$ implies that the canonical bundle $K$ is trivial on the non-singular rational curve $E_{i}$.

Let $e$ be a positive integer such that $\operatorname{dim}|e K| \geqq 0$ and let $D$ denote a pluricanonical divisor belonging to the system $|e K|$.

Lemma 4. If $D$ is a sum: $D=X+Y$ of two positive divisors $X$ and $Y$, then we have the inequality:

Proof. We let

$$
X Y \geqq 1
$$

$$
\begin{array}{ll}
X=r K+\xi, & r=K X / K^{2}, K \xi=0, \\
Y=s K+\eta, & s=K Y / K^{2}, K \eta=0,
\end{array}
$$

where $\xi$ and $\eta$ are divisorial cycles. Since $X+Y=D \approx e K$, we have a homology $: \xi+\eta \sim 0$. Hence we obtain

$$
X Y=r s K^{2}-\xi^{2} .
$$

On the other hand, $r$ and $s$ are non-negative and, since the positive divisors $X$ and $Y$ are not homologous to zero, if $\xi \sim 0$ then $r s$ is positive. If $\xi \nsim 0$, then, by Lemma $3, \xi^{2}$ is negative. Consequently, $X Y$ is a positive integer, q.e.d.

We represent the pluri-canonical divisor $D$ as a sum:

$$
D=\sum_{i=1}^{n} C_{i}=C_{1}+\cdots+C_{i}+\cdots+C_{n}
$$

of irreducible curves $C_{i}$ and let

$$
D_{i}=C_{1}+C_{2}+\cdots+C_{i} .
$$

We call the representation : $\sum_{i=1}^{n} C_{i}$ a composition series. Since $K D=e K^{2} \geqq K^{2} \geqq 1$, at least one irreducible component $\Theta$ of $D$ satisfies the inequality: $K \Theta \geqq 1$.

LEMMA 5. Let $\Theta$ be an irreducible component of $D$ with $K \Theta \geqq 1$. There exists a composition series $D=\sum_{i=1}^{n} C_{i}$ with $C_{1}=\Theta$ satisfying the condition 
$(\alpha)$

$K C_{1} \geqq 1, \quad D_{i-1} C_{i} \geqq 1$

for $i=2,3, \cdots, n$.

Proof. We choose the components $C_{2}, C_{3}, \cdots$ of $D$ successively by induction. Suppose that we have chosen $C_{1}=\Theta, C_{2}, \cdots, C_{i-1}$ such that

and let

$$
D_{j-1} C_{j} \geqq 1, \quad \text { for } j=2,3, \cdots, i-1 \text {, }
$$

$$
D=D_{i-1}+Z_{i},
$$

where $D_{j-1}=C_{1}+\cdots+C_{j-1}$. If $Z_{i}>0$, then, by Lemma $4, D_{i-1} Z_{i} \geqq 1$ and therefore at least one irreducible curve $C \leqq Z_{i}$ has $D_{i-1} C \geqq 1$. Hence, letting $C_{i}=C$, we obtain

$$
D_{i-1} C_{i} \geqq 1,
$$

q. e. d.

LEMMA 6. Let $E_{1}$ and $E_{2}$ be irreducible curves on $S$ satisfying the condition that $K E_{1}=K E_{2}=E_{1} E_{2}=0$. If $D$ is a sum:

$$
D=X+Y+E_{1}+E_{2}
$$

of $E_{1}, E_{2}$ and two positive divisors $X, Y$ and if $K X>0, K Y>0$, then $X Y$ is non-negative.

Proof. We write

$$
\begin{array}{ll}
X=r K+r_{1} E_{1}+r_{2} E_{2}+\xi, & K \xi=E_{1} \xi=E_{2} \xi=0, \\
Y=s K+s_{1} E_{1}+s_{2} E_{2}+\eta, & K \eta=E_{1} \eta=E_{2} \eta=0,
\end{array}
$$

where $\xi$ and $\eta$ are divisorial cycles. Since $E_{1}^{2}=E_{2}^{2}=-2$, the coefficients $r, s$, $r_{\nu}, s_{\nu}, \nu=1,2$, are given by the formulae:

$$
K^{2} r=K X, \quad K^{2} s=K Y, \quad-2 r_{\nu}=E_{\nu} X, \quad-2 s_{\nu}=E_{\nu} Y .
$$

The linear equivalence $X+Y+E_{1}+E_{2} \approx e K$ implies that

Hence we obtain

$$
1+r_{1}+s_{1}=0, \quad 1+r_{2}+s_{2}=0, \quad \xi+\eta \sim 0 .
$$

$$
X Y=r s K^{2}-2 r_{1} s_{1}-2 r_{2} s_{2}+\xi \eta=r s K^{2}+\sum_{n=1}^{2} 2 r_{\nu}\left(r_{\nu}+1\right)-\xi^{2} \geqq r s K^{2}-1-\xi^{2} .
$$

Since, by hypothesis, $r$ and $s$ are positive and, by Lemma $3, \xi^{2} \leqq 0$, this proves that $X Y>-1$, while $X Y$ is an integer. Consequently $X Y$ is non-negative, q. e. d.

We write the curve $\mathcal{E}=E_{1}+E_{2}+\cdots+E_{b}$ as a sum:

$$
\mathcal{E}=\mathcal{E}_{1}+\cdots+\mathcal{E}_{\nu}+\cdots+\mathcal{E}_{\kappa}
$$

of connected components $\mathcal{E}_{\nu}$. We shall say that a positive divisor $X$ meets $D$ if there exists a point $z$ such that $z \in X, z \in D$. Since $D E_{i}=e K E_{i}=0$, if $E_{i}$ meets $D$, then $E_{i}$ is a component of $D$. Hence, if $\mathcal{E}_{\nu}$ meets $D$, then $\mathcal{E}_{\nu}<D$. 
LEMMA 7. If $\mathcal{E}_{\lambda}+\mathcal{E}_{\nu}<D, \lambda \neq \nu$, then there exists a composition series $D=\sum_{i=1}^{n} C_{i}$ with $C_{n-1}<\mathcal{E}_{\lambda}, C_{n}<\mathcal{E}_{\nu}$, which satisfies the condition

$$
D_{i-1} C_{i} \geqq 0, \quad K C_{i}+D_{i-1} C_{i} \geqq 1,
$$

for $i=1,2, \cdots, n$.

Proof. We may assume that $E_{1}<\mathcal{E}_{\lambda}, E_{2}<\mathcal{E}_{\nu}$. Suppose that we have chosen $C_{1}, \cdots, C_{j}, \cdots, C_{i-1}$ satisfying

$$
D_{j-1} C_{j} \geqq 0, \quad K C_{j}+D_{j-1} C_{j} \geqq 1,
$$

in such a manner that

$$
D=D_{i-1}+X_{i}+E_{1}+E_{2}, \quad X_{i} \geqq 0,
$$

where $D_{j-1}=C_{1}+\cdots+C_{j-1}$. Then we have two alternatives: either $K X_{i}=0$ or there is an irreducible curve $C \leqq X_{i}$ satisfying the condition:

$$
D_{i-1} C \geqq 0, \quad K C+D_{i-1} C \geqq 1 .
$$

In fact, since $K D_{i-1} \geqq K C_{1} \geqq 1$, if $K X_{i}>0$, then, by Lemma $6, D_{i-1} X_{i}$ is nonnegative. It follows that either there is an irreducible curve $C \leqq X_{i}$ with $D_{i-1} C \geqq 1$ or every irreducible curve $C \leqq X_{i}$ satisfies the equation: $D_{i-1} C=0$. If $D_{i-1} C \geqq 1$ for an irreducible curve $C \leqq X_{i}$, then the curve $C$ satisfies (7). The inequality: $K X_{i}>0$ implies that an irreducible curve $C \leqq X_{i}$ satisfies $K C \geqq 1$. If $D_{i-1} C=0$, then this curve $C$ satisfies (7).

If there exists an irreducible curve $C \leqq X_{i}$ satisfying (7), then, letting: $C_{i}=C$ and $D_{i}=D_{i-1}+C_{i}$, we get

$$
D_{i-1} C_{i} \geqq 0, \quad K C_{i}+D_{i-1} C_{i} \geqq 1,
$$

and

$$
D=D_{i}+X_{i+1}+E_{1}+E_{2}, \quad X_{i+1} \geqq 0 .
$$

Thus we choose $C_{1}, \cdots, C_{i}, \cdots, C_{h}$ satisfying

$$
D_{i-1} C_{i} \geqq 0, \quad K C_{i}+D_{i-1} C_{i} \geqq 1, \quad \text { for } i=1,2, \cdots, h,
$$

where $D_{i-1}=C_{1}+\cdots+C_{i-1}$, such that

$$
D=C_{1}+\cdots+C_{h}+X+E_{1}+E_{2}, \quad X \geqq 0, K X=0 .
$$

Now, with the aid of Lemma 4, we extend the series $C_{1}+\cdots+C_{h}$ to a composition series

such that

$$
D=C_{1}+\cdots+C_{h}+C_{h+1}+\cdots+C_{n}
$$

$$
\left(C_{1}+\cdots+C_{h}+\cdots+C_{i-1}\right) C_{i} \geqq 1,
$$
for $i=h+1, \cdots, n$.

Note that $C_{i}<\mathcal{E}$ for $i=h+1, \cdots, n$. If $C_{j} C_{j+1}=0$ for an integer $j, h<j<n$, then the inequalities (8) are not affected by the permutation: $C_{j} \rightarrow C_{j+1}, C_{j+1} \rightarrow C_{j \text { a }}$ 
Moreover $E_{1}<\mathcal{E}_{\lambda}$ and $E_{2}<\mathcal{E}_{\nu}$ appear among the irreducible components $C_{i}, i$ $=h+1, \cdots, n$. Hence, by means of an appropriate permutation of the components $C_{h+1}, C_{h+2}, \cdots, C_{n}$, we obtain a composition series $D=\sum_{i=1}^{n} C_{i}$ satisfying the condition $(\beta)$ such that $C_{n-1}<\mathcal{E}_{\lambda}, C_{n}<\mathcal{E}_{\nu}$, q. e.d.

In a similar manner we obtain the following

LEMMA 8. If $\mathcal{E}_{\lambda}<D$, then there exists a composition series: $D=\sum_{i=1}^{n} C_{i}$ with $C_{n}<\mathcal{E}_{\lambda}$ which satisfies the above condition $(\beta)$.

\section{$\S 4$. Pluri-canonical systems.}

In this section we denote by $K$ the canonical bundle of $S$. Let $e$ be a positive integer such that $\operatorname{dim}|e K| \geqq 0$ and let $D$ be a member of $|e K|$. Moreover let $m$ denote an integer $>e$. For any composition series:

we let

$$
D=C_{1}+C_{2}+\cdots+C_{i}+\cdots+C_{n} \text {, }
$$

and define

$$
Z_{i}=C_{i}+C_{i+1}+\cdots+C_{n}, \quad Z_{n+1}=0,
$$

$$
F_{i}=m K-\left[Z_{i}\right] \text {. }
$$

Then, by a simple calculation, we obtain

$$
F_{i+1} C_{i}-C_{i}^{2}-K C_{i}=(m-e-1) K C_{i}+D_{i-1} C_{i} .
$$

Let $x$ and $y$ be distinct points of $D$ and let

$$
\Xi_{i}=\mathcal{O}\left(m K-Z_{i}-h x-k y\right)=\mathcal{O}\left(m K-Z_{i}\right) \cap \mathcal{O}(m K-h x-k y),
$$

where $h$ and $k$ are non-negative integers. We consider the ascending chain:

$$
\Xi_{1} \subset \Xi_{2} \subset \cdots \subset \Xi_{i} \subset \cdots \subset \Xi_{n+1}=\mathcal{O}(m K-h x-k y) .
$$

We assume that the multiplicities of the points $x$ and $y$ of $D$ are not smaller than $h$ and $k$, respectively, and that

$$
\Xi_{i+1} / \Xi_{i} \cong \mathcal{O}\left(F_{i+1}-h_{i} x-k_{i} y\right)_{C_{i}},
$$

where $h_{i}$ and $k_{i}$ are non-negative integers.

LEMMA 9. If

$$
(m-e-1) K C_{i}+D_{i-1} C_{i}>\frac{1}{4}\left(h_{i}+1\right)^{2}+\frac{1}{4}\left(k_{i}+1\right)^{2}, \quad \text { for } i=1,2, \cdots, n,
$$

then we have the inequalities

$$
\operatorname{dim} H^{1}(S, \mathcal{O}((m-e) K)) \geqq \operatorname{dim} H^{1}\left(S, \Xi_{i}\right), \quad i=2,3, \cdots, n+1 .
$$

Proof. According as $x \in C_{i}$ or $x \in C_{i}$, we define $m_{i}$ to be the multiplicity of the point $x$ of $C_{i}$ or zero. Similarly, according as $y \in C_{i}$ or $y \notin C_{i}$, we define 
$n_{i}$ to be the multiplicity of the point $y$ of $C_{i}$ or zero. Since

$$
\frac{1}{4}\left(h_{i}+1\right)^{2}+\frac{1}{4}\left(k_{i}+1\right)^{2} \geqq\left(h_{i}-m_{i}+1\right)^{+} m_{i}+\left(k_{i}-n_{i}+1\right)^{+} n_{i},
$$

we infer from Theorem 1 and the formula (9) that

$$
H^{1}\left(S, \Xi_{i+1} / \Xi_{i}\right) \cong H^{1}\left(C_{i}, \mathcal{O}\left(F_{i+1}-h_{i} x-k_{i} y\right)_{C_{i}}\right)=0 .
$$

It follows that the sequences

are exact, while

$$
H^{1}\left(S, \Xi_{i}\right) \rightarrow H^{1}\left(S, \Xi_{i+1}\right) \rightarrow 0
$$

$$
\Xi_{1}=\mathcal{O}(m K-D) \cong \mathcal{O}((m-e) K) .
$$

Hence we obtain the inequalities (10), q.e.d.

Lemma 10. There exists an integer $m_{0}$ such that

$$
\operatorname{dim} H^{1}(S, \mathcal{O}((m-e) K))=\operatorname{dim} H^{1}(S, \mathcal{O}(m K)),
$$

for $m \geqq m_{0}$.

(see Zariski [9]).

Proof. With the aid of Lemma 5, we choose a composition series: $D=\sum_{i=1}^{n} C_{i}$ satisfying the condition $(\alpha)$ and let

We have

$$
\Xi_{i}=\mathcal{O}\left(m K-Z_{i}\right)
$$

$$
\Xi_{i+1} / \Xi_{i} \cong \mathcal{O}\left(F_{i+1}\right) / \mathcal{O}\left(F_{i+1}-C_{i}\right)=\mathcal{O}\left(F_{i+1}\right)_{c_{i}} .
$$

Assume that $m \geqq e+2$. Then it follows from the condition $(\alpha)$ that

$$
(m-e-1) K C_{i}+D_{i-1} C_{i} \geqq 1 \text {. }
$$

Hence, by Lemma 9, we have the inequality

$$
\operatorname{dim} H^{1}(S, \mathcal{O}((m-e) K)) \geqq \operatorname{dim} H^{1}(S, \mathcal{O}(m K)) .
$$

Hence we infer readily the existence of an integer $m_{0}$ such that the equality (11) holds for $m \geqq m_{0}$, q. e. d.

For any point $x \in S$, we have the exact sequence

$$
0 \rightarrow \mathcal{O}(m K-x) \rightarrow \mathcal{O}(m K) \rightarrow \boldsymbol{C}_{x} \rightarrow 0
$$

(see (1)) and the corresponding exact cohomology sequence

$$
\begin{aligned}
0 & \rightarrow H^{0}(S, \mathcal{O}(m K-x)) \rightarrow H^{0}(S, \mathcal{O}(m K)) \rightarrow \boldsymbol{C} \\
& \rightarrow H^{1}(S, \mathcal{O}(m K-x)) \rightarrow H^{1}(S, \mathcal{O}(m K)) \rightarrow 0 \rightarrow \cdots .
\end{aligned}
$$

THEOREM 4. Let $e$ be a positive integer such that $P_{e} \geqq 2, e K^{2} \geqq 2$. तIf. $m \geqq e+2$ and if $m \geqq m_{0}$, then, for every point $x \in S$, the sequence

$$
0 \rightarrow H^{0}(S, \mathcal{O}(m K-x)) \rightarrow H^{0}(S, \mathcal{O}(m K)) \rightarrow \boldsymbol{C} \rightarrow 0
$$


is exact.

PRoof. Since $\operatorname{dim}|e K|=P_{e}-1 \geqq 1$, we find a divisor $D \in|e K|$ such that $x \in D$.

I) The case in which $x \notin \mathcal{E}$. We choose a composition series: $D=\sum_{i=1}^{n} C_{i}$ satisfying the condition $(\alpha)$ and let

$$
\boldsymbol{\Xi}_{i}=\mathcal{O}\left(m K-Z_{i}-x\right) \text {. }
$$

We find an integer $h$ such that $x \in C_{h}, x \notin Z_{h+1}$. Since $C_{h} \nless \mathcal{E}$, we have $K C_{h} \geqq 1$. Moreover, we may assume that $K C_{h} \geqq 2$ if $h=1$. In fact, since, by hypothesis,

$$
K D=e K^{2} \geqq 2 \text {, }
$$

if $K C_{h}=1$, then there exists an irreducible curve $\Theta \leqq D-C_{h}$ with $K \Theta \geqq 1$. In view of Lemma 5 , we may assume that $C_{1}=\Theta$. It follows that $h \geqq 2$.

Since $x \in Z_{i}$ for $i \leqq h$, we have

$$
\Xi_{i}=\mathcal{O}\left(m K-Z_{i}\right)
$$

for $i \leqq h$.

We have the commutative diagram:

$$
\begin{aligned}
& \mathcal{O}\left(m K-Z_{h}\right) \subseteq \mathcal{O}\left(m K-Z_{h+1}-x\right) \\
& \text { থ॥ } 2 \text { | } \\
& \mathcal{O}\left(F_{h+1}-C_{h}\right) \subseteq \mathcal{O}\left(F_{h+1}-x\right) .
\end{aligned}
$$

Hence we obtain the isomorphism:

Thus we infer that

$$
\Xi_{h+1} / \Xi_{h} \cong \mathcal{O}\left(F_{h+1}-x\right)_{c_{h}}
$$

$$
\Xi_{i+1} / \Xi_{i} \cong \mathcal{O}\left(F_{i+1}-\delta_{i h} x\right)_{C_{i}},
$$

where $\delta_{i h}$ denotes Kronecker's delta. Since $m-e \geqq 2$ and $K C_{h} \geqq 1+\delta_{h 1}$, it follows from the condition $(\alpha)$ that

$$
(m-e-1) K C_{i}+D_{i-1} C_{i} \geqq 1+\delta_{i n} .
$$

Hence, by Lemma 9, we have the inequality

$$
\operatorname{dim} H^{1}(S, \mathcal{O}((m-e) K)) \geqq \operatorname{dim} H^{1}(S, \mathcal{O}(m K-x)) .
$$

Combining this with (11) and (12), we infer the exactness of (13),

II) The case in which $x \in \mathcal{E}_{\lambda}$. With the aid of Lemma 8, we choose a composition series: $D=\sum_{i=1}^{n} C_{i}$ with $C_{n}<\mathcal{E}_{\lambda}$ which satisfies the condition ( $\beta$ ) and let

Since $\Xi_{i+1} / \Xi_{i} \cong \mathcal{O}\left(F_{i+1}\right)_{C_{i}}$ and

$$
\Xi_{i}=\mathcal{O}\left(m K-Z_{i}\right)
$$

$$
(m-e-1) K C_{i}+D_{i-1} C_{i} \geqq K C_{i}+D_{i-1} C_{i} \geqq 1,
$$

we have, by Lemma 9, 


$$
\operatorname{dim} H^{1}(S, \mathcal{O}((m-e) K)) \geqq \operatorname{dim} H^{1}\left(S, \boldsymbol{\Xi}_{n}\right) .
$$

Combined with (11), this proves that

$$
\operatorname{dim} H^{1}(S, \mathcal{O}(m K)) \geqq \operatorname{dim} H^{1}\left(S, \mathcal{O}\left(m K-C_{n}\right)\right) .
$$

Since $K$ is trivial on $C_{n}$, we have the exact sequence

$$
0 \rightarrow \mathcal{O}\left(m K-C_{n}\right) \rightarrow \mathcal{O}(m K) \rightarrow \mathcal{O}_{C_{n}} \rightarrow 0 .
$$

Moreover $C_{n}$ is a non-singular rational curve. Hence we obtain the exact sequence

$$
\begin{aligned}
0 & \rightarrow H^{0}\left(S, \mathcal{O}\left(m K-C_{n}\right)\right) \rightarrow H^{0}(S, \mathcal{O}(m K)) \rightarrow C \\
& \rightarrow H^{1}\left(S, \mathcal{O}\left(m K-C_{n}\right)\right) \rightarrow H^{1}(S, \mathcal{O}(m K)) \rightarrow 0 .
\end{aligned}
$$

Combining this with (14), we infer that the sequence

$$
0 \rightarrow H^{0}\left(S, \mathcal{O}\left(m K-C_{n}\right)\right) \rightarrow H^{0}(S, \mathcal{O}(m K)) \rightarrow C \rightarrow 0
$$

is exact, while every holomorphic section $\varphi \in H^{0}(S, \mathcal{O}(m K))$ is reduced to a constant on $\mathcal{E}_{\lambda}$. Hence the exactness of (13) follows.

THEOREM 5. The cohomology group $H^{1}(S, \mathcal{O}(m K))$ vanishes for every integer $m \geqq 2$.

Proof. Let $e$ be a positive integer such that $P_{e} \geqq 2, e K^{2} \geqq 2$. The existence of such an integer $e$ is obvious by the Riemann-Rock theorem. Let $k=m-1$ and choose a positive integer $n$ such that $n k \geqq e+2+m_{0}$. By Theorem 4 , the sequence

$$
0 \rightarrow H^{0}(S, \mathcal{O}(n k K-x)) \rightarrow H^{0}(S, \mathcal{O}(n k K)) \rightarrow \boldsymbol{C} \rightarrow 0
$$

is exact for every point $x \in S$. It follows that the complete linear system $|n k K|$ has no base point, while $(k K)^{2}=k^{2} K^{2}>0$. Hence, by Theorem 2,

q. e. d.

$$
H^{1}(S, \mathcal{O}(m K))=H^{1}(S, \mathcal{O}(k K+K))=0,
$$

Corollary. The pluri-genera $P_{m}, m \geqq 2$, are given by the formula:

$$
P_{m}=\frac{1}{2} m(m-1) K^{2}+p_{g}-q+1 \text {. }
$$

THEOREM 6. Let $e$ be a positive integer such that $P_{e} \geqq 2, e K^{2} \geqq 2$. If $m \geqq e+2$, then the pluri-canonical system $|m K|$ has no base point and the map $\Phi_{m K}$ is holomorphic.

PROof. It follows from Theorem 5 that $m_{0}=e+2$, where $m_{0}$ is the integer appeared in (11). Hence we infer from Theorem 4 that, if $m \geqq e+2$, then $|m K|$ has no base point and, consequently, $\Phi_{m K}$ is a holomorphic map, q.e.d.

For any pair of distinct points $x$ and $y$ on $S$, we have the exact sequence

$$
0 \rightarrow \mathcal{O}(m K-x-y) \rightarrow \mathcal{O}(m K) \rightarrow \boldsymbol{C}_{x} \oplus \boldsymbol{C}_{y} \rightarrow 0
$$


(see (1)) and the corresponding exact cohomology sequence

$$
\cdots \rightarrow H^{0}(S, \mathcal{O}(m K)) \rightarrow C^{2} \rightarrow H^{1}(S, \mathcal{O}(m K-x-y)) \rightarrow \cdots .
$$

We shall say that $x$ and $y$ are distinct modulo $\mathcal{E}$ if $x$ and $y$ are distinct and not contained in one and the same connected component of $\varepsilon$.

THEOREM 7. Let $e$ be a positive integer such that $P_{e} \geqq 3, e K^{2} \geqq 2$. If $m \geqq e+3$, then, for any pair of points $x$ and $y$ on $S$ which are distinct modulo $\mathcal{E}$, the sequence

$$
0 \rightarrow H^{0}(S, \mathcal{O}(m K-x-y)) \rightarrow H^{0}(S, \mathcal{O}(m K)) \rightarrow C^{2} \rightarrow 0
$$

is exact.

Proof. Since $\operatorname{dim}|e K|=P_{e}-1 \geqq 2$, we find a divisor $D \in|e K|$ such that $x \in D, y \in D$.

I) The case in which $x, y \in \mathcal{E}$. With the aid of Lemma 5 , we choose a composition series: $D=\sum_{i=1}^{n} C_{i}$ satisfying the condition $(\alpha)$ and let

$$
\Xi_{i}=\mathcal{O}\left(m K-Z_{i}-x-y\right) \text {. }
$$

We find $h$ and $j$ such that $x \in C_{h}, x \notin Z_{h+1}, y \in C_{j}, y \notin Z_{j+1}$. Then we have

$$
\Xi_{i+1} / \Xi_{i} \cong \mathcal{O}\left(F_{i+1}-\delta_{i n} x-\delta_{i j} y\right)_{C_{i}} .
$$

Since $C_{h} \varangle \mathcal{E}, C_{j} \varangle \mathcal{E}$, we have $K C_{h} \geqq 1, K C_{j} \geqq 1$ and, as was mentioned in the proof of Theorem 4 , we may assume that $K C_{1} \geqq 2$ if $h$ is equal to 1 . The condition $(\alpha)$ implies therefore that

$$
(m-e-1) K C_{i}+D_{i-1} C_{i} \geqq 1+\delta_{i h}+\delta_{i j} .
$$

Hence, by Lemma 9 and Theorem $5, H^{1}\left(S, \Xi_{n+1}\right)$ vanishes. It follows that the sequence (16) is exact.

II) The case in which $x \in \mathcal{E}_{\lambda}, y \in \mathcal{E}_{\nu}, \lambda \neq \nu$. With the aid of Lemma 7, we choose a composition series: $D=\sum_{i=1}^{n} C_{i}$ with $C_{n-1}<\mathcal{E}_{\lambda}, C_{n}<\mathcal{E}_{\nu}$ which satisfies the condition $(\beta)$ and let

$$
\Xi_{i}=\mathcal{O}\left(m K-Z_{i}\right)
$$

Since $\Xi_{i+1} / \Xi_{i} \cong \mathcal{O}\left(F_{i+1}\right)_{C_{i}}$ and

$$
(m-e-1) K C_{i}+D_{i-1} C_{i} \geqq K C_{i}+D_{i-1} C_{i} \geqq 1,
$$

we infer from Lemma 9 and Theorem 5 that

$$
H^{1}\left(S, \mathcal{O}\left(m K-C_{n-1}-C_{n}\right)\right)=0 .
$$

Since $K$ is trivial on $C_{n-1}$ and on $C_{n}$, we have the exact sequence

$$
0 \rightarrow \mathcal{O}\left(m K-C_{n-1}-C_{n}\right) \rightarrow \mathcal{O}(m K) \rightarrow \mathcal{O}_{C_{n-1}} \oplus \mathcal{O}_{C_{n}} \rightarrow 0 .
$$

Combining this with (17), we infer that the sequence 


$$
0 \rightarrow H^{0}\left(S, \mathcal{O}\left(m K-C_{n-1}-C_{n}\right)\right) \rightarrow H^{0}(S, \mathcal{O}(m K)) \rightarrow \boldsymbol{C}^{2} \rightarrow 0
$$

is exact, while every holomorphic section $\varphi \in H^{0}(S, \mathcal{O}(m K))$ is reduced to a constant on each connected component of $\mathcal{E}$. Hence the exactness of (16) follows.

III) The case in which $x \notin \mathcal{E}, y \in \mathcal{E}_{\lambda}$. We choose a composition series: $D=\sum_{i=1}^{n} C_{i}$ with $C_{n}<\varepsilon_{\lambda}$ satisfying the condition $(\beta)$ and let

$$
\Xi_{i}=\mathcal{O}\left(m K-Z_{i}-x\right) \text {. }
$$

We find $h$ such that $x \in C_{h}, x \notin Z_{h+1}$. Then we have

$$
\Xi_{i+1} / \Xi_{i} \cong \mathcal{O}\left(F_{i+1}-\delta_{i h} x\right)_{C_{i}} \text {. }
$$

Moreover, since $K C_{h} \geqq 1$, we have

$$
(m-e-1) K C_{i}+D_{i-1} C_{i} \geqq 1+\delta_{i n} .
$$

Hence, by Lemma 9 and Theorem 5, we get

$$
H^{1}\left(S, \mathcal{O}\left(m K-C_{n}-x\right)\right)=0 .
$$

Combining this with the exact sequence

$$
0 \rightarrow \mathcal{O}\left(m K-C_{n}-x\right) \rightarrow \mathcal{O}(m K) \rightarrow \mathcal{O}_{C_{n}} \oplus \boldsymbol{C}_{x} \rightarrow 0,
$$

we infer that the sequence

$$
0 \rightarrow H^{0}\left(S, \mathcal{O}\left(m K-C_{n}-x\right)\right) \rightarrow H^{0}(S, \mathcal{O}(m K)) \rightarrow C^{2} \rightarrow 0
$$

is exact. Hence the exactness of (16) follows, q. e.d.

Now we consider the exact sequence

$$
0 \rightarrow \mathcal{O}(m K-2 x) \rightarrow \mathcal{O}(m K) \rightarrow \boldsymbol{C}_{\boldsymbol{x}}^{\boldsymbol{s}} \rightarrow 0 .
$$

THEOREM 8. Let $e$ be a positive integer such that $P_{e} \geqq 4, e K^{2} \geqq 2$. If $m \geqq e+3$ and if $x \in \mathcal{E}$, then the sequence

$$
0 \rightarrow H^{0}(S, \mathcal{O}(m K-2 x)) \rightarrow H^{0}(S, \mathcal{O}(m K)) \rightarrow \boldsymbol{C}^{\mathbf{8}} \rightarrow 0
$$

is exact.

Proof. Since, by hypothesis, $\operatorname{dim}|e K|=P_{e}-1 \geqq 3$, we find a divisor $D \in|e K|$ such that $x$ is a multiple point of $D$. We choose a composition series: $D=\sum_{i=1}^{n} C_{i}$ satisfying the condition $(\alpha)$ and let

$$
\Xi_{i}=\mathcal{O}\left(m K-Z_{i}-2 x\right) .
$$

We find $h$ such that $x \in C_{h}, x \in Z_{h+1}$. As was mentioned in the proof of Theorem 4 , we may assume that $K C_{h} \geqq 2$ if $h=1$. To prove the exactness of (18) it suffices to show the vanishing of $H^{1}\left(S, \Xi_{n+1}\right)$.

i) If $x$ is a multiple point of $C_{h}$, then 
and therefore

$$
\Xi_{i}=\mathcal{O}\left(m K-Z_{i}\right), \quad \text { for } i \leqq h,
$$

$$
\Xi_{i+1} / E_{i} \cong \mathcal{O}\left(F_{i+1}-2 \delta_{i n} x\right)_{C_{i}} .
$$

Since $m-e \geqq 3$ and $K C_{1} \geqq 1+\delta_{h 1}$, it follows from the condition $(\alpha)$ that

$$
(m-e-1) K C_{i}+D_{i-1} C_{i} \geqq 1+2 \delta_{i n} .
$$

Hence, by Lemma 9 and Theorem $5, H^{1}\left(S, \boldsymbol{E}_{n+1}\right)$ vanishes.

ii) If $x$ is a simple point of $C_{h}$, then we find an integer $j<h$ such that

$$
x \in C_{j}, \quad x \in C_{j+1}+C_{j+2}+\cdots+C_{h-1} .
$$

Since $x$ is a simple point of $Z_{j+1}$, we have the isomorphism:

$$
\mathcal{O}\left(m K-Z_{j+1}-2 x\right) \cong \mathcal{O}\left(F_{j+1}-x\right) \text {. }
$$

Moreover we have the commutative diagram:

$$
\begin{aligned}
& \mathcal{O}\left(m K-Z_{j}\right) \subseteq \mathcal{O}\left(m K-Z_{j+1}-2 x\right) \\
& \quad 2 \| \quad \text { थ\| } \\
& \mathcal{O}\left(F_{j+1}-C_{j}\right) \subseteq \mathcal{O}\left(F_{j+1}-x\right) .
\end{aligned}
$$

Hence $\Xi_{j+1} / \Xi_{j}$ is isomorphic to $\mathcal{O}\left(F_{j+1}-x\right)_{c_{j}}$. Thus we see that

$$
\Xi_{i+1} / \Xi_{i} \cong \mathcal{O}\left(F_{i+1}-\left(\delta_{i j}+2 \delta_{i n}\right) x\right)_{c_{i}} .
$$

Moreover, since $K C_{j} \geqq 1, K C_{h} \geqq 1$, the condition $(\alpha)$ implies that

$$
(m-e-1) K C_{i}+D_{i-1} C_{i} \geqq 1+\delta_{i j}+2 \delta_{i h} .
$$

Hence, by Lemma 9 and Theorem $5, H^{1}\left(S, \Xi_{n+1}\right)$ vanishes, q.e.d.

Let $\Phi$ be a holomorphic map of $S$ into a complex manifold. We shall say that $\Phi$ is one-to-one modulo $\mathcal{E}$ if any only if

$$
\Phi^{-1} \Phi(z)=\left\{\begin{array}{lr}
z, & \text { for } z \in S-\mathcal{E}, \\
\varepsilon_{\lambda} & \text { for } z \in \mathcal{E}_{\lambda} .
\end{array}\right.
$$

Moreover we say that $\Phi$ is biholomorphic modulo $\mathcal{E}$ if $\Phi$ is one-to-one modulo $\mathcal{E}$ and biholomorphic on $S-\mathcal{E}$.

THEOREM 9. Let $e$ be a positive integer such that $P_{e} \geqq 3, e K^{2} \geqq 2$. For every integer $m \geqq e+3$, the map $\Phi_{m K}$ is holomorphic and one-to-one modulo $\mathcal{E}$.

Proof. We infer from Theorem 7 that $\Phi_{m K}$ is holomorphic and $\Phi_{m K}(x)$ $\neq \Phi_{m K}(y)$ for any pair of points $x, y$ on $S$ which are distinct modulo $\mathcal{E}$. Moreover the image $\Phi_{m K}\left(\mathcal{E}_{\lambda}\right)$ of each component $\mathcal{E}_{\lambda}$ is a point, since $K$ is trivial on $\mathcal{E}_{\lambda}$, q. e.d.

The exactness of the sequence (18) implies that $\Phi_{m K}$ is biholomorphic in a neighborhood of $x$ on $S$. Hence we infer from Theorems 8 and 9 the following

THEOREM 10. Let $e$ be a positive integer such that $P_{e} \geqq 4, e K^{2} \geqq 2$. For 
every integer $m \geqq e+3$, the map $\Phi_{m K}$ is holomorphic and biholomorphic modulo $\mathcal{E}$. LEMMA 11. If $p_{g}=0$, then $q=0$.

Proof. We have the Noether formula:

$$
8 q+K^{2}+b_{2}=12 p_{g}+10,
$$

where $b_{2}$ denotes the second Betti number of $S$. Since $K^{2} \geqq 1$, this formula proves that, if $p_{g}=0$, then $q \leqq 1$. Suppose that $q=1$. Then there exists a holomorphic map $\Psi$ of $S$ onto an elliptic curve $\Delta$ such that the inverse image $C=\Psi^{-1}(u)$ of any general point $u \in \Delta$ is an irreducible non-singular curve. Since $C^{2}=0, C$ and $K$ are homologically independent. It follows that $b_{2} \geqq 2$. This contradicts the Noether formula. Thus we infer that $q=0$, q.e.d.

LemMA 12. If $K^{2}=1$, then $p_{g} \leqq 2$ and $q \leqq 1$.

Proof. i) Assume that $p_{g} \geqq 2$. Any general member of $|K|$ is an irreducible non-singular curve of genus 2. To prove this we let $D$ denote a general member of $|K|$. The general member $D$ has an irreducible component $C$ with $C^{2} \geqq 0$. Since $K D=K^{2}=1$, we have

while

$$
D=C+X, \quad X \geqq 0, \quad K C=1, \quad K X=0,
$$

$$
C^{2}=2 \pi(C)-2-K C, \quad C^{2}+C X=K C .
$$

Hence we infer that $C X=0$ and therefore, by Lemma $4, X=0$. Thus we see that $D=C$. If follows that $\pi(C)=2$. By a theorem of Bertini, $C$ has no singular point outside the base points of $|K|$, while, since $C^{2}=1$, any base point of $|K|$ is a simple point of $C$. Hence $C$ is a non-singular curve. It is clear that

$$
\operatorname{dim} H^{0}\left(C, \mathcal{O}([C])_{C}\right) \leqq 1
$$

Combining this with the exact sequence

$$
0 \rightarrow H^{0}(S, \mathcal{O}) \rightarrow H^{0}(S, \mathcal{O}(C)) \rightarrow H^{0}\left(C, \mathcal{O}([C])_{C}\right) \rightarrow \cdots
$$

we obtain the inequality

$$
p_{g}=\operatorname{dim} H^{0}(S, \mathcal{O}(C)) \leqq 2 .
$$

ii) Since $P_{2} \geqq p_{g}$, we infer from (15) that

$$
q=K^{2}+1+p_{g}-P_{2} \leqq 2 .
$$

iii) Now we assume that $q=2$ and derive a contradiction. There exist on $S$ two linearly independent holomorphic 1-forms $\varphi_{1}$ and $\varphi_{2}$.

If $\varphi_{1} \wedge \varphi_{2}=0$, then there exists a holomorphic map $\Psi$ of $S$ onto a nonsingular algebraic curve $\Delta$ of genus 2 such that the inverse image $\Theta_{u}=\Psi^{-1}(u)$ of any general point $u \in \Delta$ is an irreducible non-singular curve. Since, by Lemma $11, p_{g} \geqq 1$, the canonical system $|K|$ contains a positive divisor $D$. 
Since $K D=K^{2}=1$, we have a composition series :

$$
D=C+\sum_{i=2}^{n} E_{i}, \quad K C=1, K E_{i}=0 .
$$

Since $\Theta_{u}^{2}=0, K \Theta_{u}$ is positive, while $K \Theta_{u}=2 \pi\left(\Theta_{u}\right)-2$ is even. Moreover the projection $\Psi\left(E_{i}\right)$ of each rational curve $E_{i}$ is a point on $\Delta$. Hence $C \Theta_{u}=K \Theta_{u} \geqq 2$ and therefore $C$ is a covering of $\Delta$ with at least two sheets. It follows that

This contradicts that

$$
2 \pi(C)-2 \geqq 4 \pi(\Delta)-4 \geqq 4 \text {. }
$$

$$
2 \pi(C)-2=C^{2}+K C=2 K C-\sum_{i} C E_{i} \leqq 2 .
$$

If $\varphi_{1} \wedge \varphi_{2} \neq 0$, then $\varphi_{1}$ and $\varphi_{2}$ define a holomorphic map $\Phi$ of $S$ onto the Albanese variety $A$ attached to $S$. The canonical divisor $\left(\varphi_{1} \wedge \varphi_{2}\right)$ is an irreducible non-singular curve of genus 2. To prove this we let

$$
\left(\varphi_{1} \wedge \varphi_{2}\right)=C+X, \quad X \geqq 0, K C=1, K X=0 .
$$

Suppose that the restrictions $\varphi_{1 C}$ and $\varphi_{2 C}$ of $\varphi_{1}$ and $\varphi_{2}$ to $C$ are linearly dependent. Then $\Phi(C)$ is either a point or an elliptic curve on $A$. If $X>0$, then $X$ is composed of non-singular rational curves $E_{i}<\varepsilon$. Hence $\Phi(X)$ consists of a finite number of points on $A$. Consequently, there exists an irreducible nonsingular curve $\Gamma$ on $A$ which meets neither $\Phi(C)$ nor $\Phi(X)$. It follows that $K \Phi^{-1}(\Gamma)=0$ and therefore $\Phi^{-1}(\Gamma)$ is composed of rational curves. This contradicts that $\pi(\Gamma) \geqq 1$.

Thus $\varphi_{1 C}$ and $\varphi_{2 C}$ are linearly independent and therefore the genus of the non-singular model of $C$ is not smaller than 2 , while

$$
2 \pi(C)-2=C^{2}+K C=2-C X
$$

and, by Lemma 4, $C X$ is positive if $X>0$. Hence we infer that $C$ is a nonsingular curve of genus 2 and $X=0$. It follows that $\left(\varphi_{1} \wedge \varphi_{2}\right)=C$.

The Euler number of $S$ is equal to the sum of the indices of the singular points of the covariant vector field $\varphi_{1}$. Since $\left(\varphi_{1} \wedge \varphi_{2}\right)=C$, the vector field $\varphi_{1}$ has no singular point outside $C$. We may assume that $\varphi_{1 C}$ has two simple zeros $x$ and $y$ on $C$. Since $\varphi_{2 C}$ does not vanish at $x$, we can choose a local coordinate $(w, z)$ of the center $x$ on $S$ such that

It follows that

$$
\varphi_{2}=d z, \quad \varphi_{1} \wedge \varphi_{2}=w d w \wedge d z .
$$

$$
\varphi_{1}=w d w+\rho z d z, \quad \rho \neq 0,
$$

where $\rho$ is a holomorphic function of $z$. This shows that $x$ is a singular point of $\varphi_{1}$ of index 1 . Thus the vector field $\varphi_{1}$ has exactly two singular points of index 1 and therefore the Euler number $\chi(S)$ of $S$ is equal to 2. This con- 
tradicts the Noether formula:

q.e.d.

$$
\chi(S)+K^{2}=12\left(p_{g}-q+1\right)
$$

THEOREM 11. The bigenus of $S$ is not smaller than two: $P_{2} \geqq 2$.

PROOF. i) If $p_{g} \geqq 2$, then it is obvious that $P_{2} \geqq p_{g} \geqq 2$.

ii) If $p_{g}=1$, then, by the Noether formula, $q \leqq 2$. If, moreover, $K^{2}=1$, then, by Lemma $12, q \leqq 1$. Hence, using (15), we obtain

$$
P_{2}=K^{2}+p_{g}-q+1 \geqq 2 \text {. }
$$

iii) If $p_{g}=0$, then, by Lemma $11, q=0$ and therefore

$$
P_{2}=K^{2}+1 \geqq 2 \text {. }
$$

Thus we see that $P_{2} \geqq 2$. Moreover, using (15), we get

$$
P_{3}=2 K^{2}+P_{2} \geqq 4 \text {. }
$$

Hence we infer from Theorems 6 and 10 the following

THEOREM 12. For every integer $m \geqq 4$, the pluricanonical system $|m K|$ has no base point and the map $\Phi_{m K}$ is holomorphic. For every integer $m \geqq 6$, the map $\Phi_{m K}$ is holomorphic and biholomorphic modulo $\mathcal{E}$.

If $p_{g} \geqq 4$, then, by Lemma $12, K^{2} \geqq 2$. Hence we infer from Theorem 10 the following

THEOREM 13. If $p_{g} \geqq 4$, then, for every integer $m \geqq 4$, the map $\Phi_{m K}$ is holomorphic and biholomorphic modulo $\mathcal{E}$.

\section{$\S 5$. Birational embeddings.}

It has been shown by Šafarevič [8] that, if $p_{g} \geqq 4$, then $\Phi_{3 K}$ is a birational map. In this section we prove in the context of this paper that, if $p_{g} \geqq 4$, then $|3 K|$ has no base point and $\Phi_{3 K}$ is a holomorphic birational map.

Let $\Lambda$ denote the set of those irreducible curves $C$ on $S$ which satisfy the inequality : $K C \leqq 1$.

LEMMA 13. If $K^{2} \geqq 2$, then $\Lambda$ is a finite set.

Proof. In view of Theorem 3 it suffices to consider the subset $\Lambda_{1}$ of $\Lambda$ consisting of irreducible curves $C$ with $K C=1$. We choose a base $\left\{K, B_{1}, \cdots\right.$, $\left.B_{i}, \cdots, B_{h}\right\}$ of divisorial cycles on $S$ such that $B_{1}, \cdots, B_{i}, \cdots$ are divisors satisfying the conditions

$$
B_{i}^{2}<0, \quad K B_{i}=0, \quad B_{i} B_{k}=0 \quad \text { for } i \neq k .
$$

For each curve $C \in \Lambda_{1}$, we have a homology

$$
C \sim r_{0} K+\sum_{i=1}^{h} r_{i} B_{i}, \quad r_{0}=1 / K^{2}, \quad r_{i}=B_{i} C / B_{i}^{2} .
$$


We have

$$
C^{2}=1 / K^{2}+\sum_{i=1}^{n} r_{i}^{2} B_{i}^{2} \leqq 1 / K^{2} \leqq 1 / 2
$$

and

$$
C^{2}=2 \pi(C)-2-K C=2 \pi(C)-3 .
$$

Hence we infer that $C^{2}=-1$ or -3 and that

$$
-\sum_{i=1}^{n} r_{i}^{2} B_{i}^{2}<4
$$

The homology class of $C$ contains no irreducible curve other than $C$. In fact, if $\Theta$ is an irreducible curve on $S$ and if $\Theta \sim C$, then $\Theta C=C^{2}<0$ and therefore $\Theta$ coincides with $C$. Moreover $r_{i} B_{i}^{2}=B_{i} C, i=1,2, \cdots, h$, are rational integers. Hence we infer from (19) the finiteness of the set $\Lambda_{1}$, q. e. d.

THEOREM 14. If $p_{g} \geqq 4$, then the tri-canonical system $|3 K|$ has no base point and $\Phi_{3 R}$ is a holomorphic birational map.

Proof. Since, by hypothesis, $p_{g} \geqq 4$, we have, by Lemma $12, K^{2} \geqq 2$. Hence, by Theorem 6 , the tri-canonical system $|3 K|$ has no base point and $\Phi_{3 K}$ is a holomorphic map. Moreover, by Lemma $13, \Lambda$ is a finite set. Let $\mathcal{C}$ denote the union of the curves $C \in \Lambda$. To prove that $\Phi_{3 K}$ is a birational map, it suffices to show that, for any pair of distinct points $x, y \in S-\mathcal{C}$, the sequence

$$
0 \rightarrow H^{0}(S, \mathcal{O}(3 K-x-y)) \rightarrow H^{0}(S, \mathcal{O}(3 K)) \rightarrow \boldsymbol{C}^{2} \rightarrow 0
$$

is exact.

We denote by $|K-x-y|$ the linear subsystem of $|K|$ consisting of those divisors $D \in|K|$ which pass through $x$ and $y$ in the sense that $x \in D, y \in D$. It is obvious that

$$
\operatorname{dim}|K-x-y| \geqq p_{g}-3 \geqq 1 \text {. }
$$

Let $D$ be a general member of $|K-x-y|$. We choose a composition series: $D=\sum_{i=1}^{n} C_{i}$ satisfying the condition $(\alpha)$ and let

$$
\Xi_{i}=\mathcal{O}\left(3 K-Z_{i}-x-y\right) \text {. }
$$

We find $h$ and $j$ such that $x \in C_{h}, x \in Z_{h+1}, y \in C_{j}, y \notin Z_{j+1}$. Since, by hypothesis, $x \notin \mathcal{C}, y \notin \mathcal{C}$, we have $K C_{h} \geqq 2, K C_{j} \geqq 2$. Moreover, we may assume that $K C_{1} \geqq 3$ if $h=j=1$. To show this we suppose that $h=j=1$ for every composition series: $D=\sum_{i=1}^{n} C_{i}$ satisfying the condition ( $\alpha$ ). Then, in view of Lemma $5, K C_{i}$ vanishes for $i \geqq 2$. Thus the composition series has the form

$$
D=C+E_{2}+\cdots+E_{i}+\cdots+E_{n}, \quad E_{i}<\mathcal{\varepsilon},
$$

where $C=C_{1}$. Since $D$ is a general member of $|K-x-y|$ and since $E_{i}^{2}=-2$, 
the sum: $\sum_{i=2}^{n} E_{i}$ is the fixed component of $|K-x-y|$. Let $C^{\prime}+\sum_{i=2}^{n} E_{i}$ be another general member of $|K-x-y|$. Then $C$ and $C^{\prime}$ intersect at $x$ and $y$ and therefore

$$
K C=C^{2}+\sum_{i=2}^{n} E_{i} C \geqq C^{2}=C C^{\prime} \geqq 2 .
$$

Suppose that $K C=2$. Then $C^{2}=C C^{\prime}=2$, and, by Lemma 4, $D=C$. It follows that $C \cap C^{\prime}=x \cup y$. By a theorem of Bertini, the general member $C$ has no singular point outside the base points $x$ and $y$, while, since $C C^{\prime}=2, x$ and $y$ are simple points of $C$. Thus $C$ is a non-singular curve. It is clear that $\pi(C)$ $=3$. Thus $C$ is non-rational and therefore

$$
\operatorname{dim} H^{0}\left(C, \mathcal{O}(K)_{C}\right) \leqq K C=2 .
$$

Since, by hypothesis, $p_{g} \geqq 4$, this contradicts the exact sequence

$$
0 \rightarrow \boldsymbol{C} \rightarrow H^{0}(S, \mathcal{O}(K)) \rightarrow H^{0}\left(C, \mathcal{O}(K)_{C}\right) \rightarrow \cdots
$$

Thus we see that $K C_{1} \geqq 3$.

We have

$$
\Xi_{i+1} / \Xi_{i} \cong \mathcal{O}\left(3 K-\delta_{i h} x-\delta_{i j} y\right)_{C_{i}} .
$$

Since $K C_{h} \geqq 2, K C_{j} \geqq 2$ and $K C_{1} \geqq 3$ if $h=j=1$, the condition ( $\alpha$ ) implies that

$$
K C_{i}+D_{i-1} C_{i} \geqq 1+\delta_{i n}+\delta_{i j} .
$$

Hence, by Lemma 9 and Theorem 5, $H^{1}\left(S, \Xi_{n+1}\right)$ vanishes and, consequently, the sequence (20) is exact, q. e. d.

University of Tokyo

\section{References}

[1] F. Enriques, Le Superficie Algebriche, Bologna, 1949.

[2] K. Kodaira, On compact complex analytic surfaces, I, Ann. of Math., 71 (1960), 111-152.

[3] K. Kodaira, On a differential geometric method in the theory of analytic stacks, Proc. Nat. Acad. Sci. U.S. A., 39 (1953), 1268-1273.

[4] K. Kodaira, On the structure of compact complex analytic surfaces, IV, to appear in Amer. J. Math.

[5] T. Matsusaka and D. Mumford, Two fundamental theorems on deformations of polarized varieties, Amer. J. Math., 86 (1964), 668-684.

[6] D. Mumford, The canonical ring of an algebraic surface, Ann. of Math., 76; (1962), 612-615.

[7] D. Mumford, Pathologies III, Amer. J. Math., 89 (1967), 94-104.

[8] I. P. Šafarevič, Algebraic Surfaces, Moskva, 1965.

[9] O. Zariski, The theorem of Riemann-Roch for high multiples of an effective: divisor on an algebraic surface, Ann. of Math., 76 (1962), 560-612. 